\title{
Nature of Singularity Formed by the Gravitational Collapse in Husain Space-Time with Electromagnetic Field and Scalar Field
}

\author{
Ujjal Debnath 1 * \\ Prabir Rudra $2_{*}^{*}$ \\ Ritabrata Biswas 3 t \\ *Department of Mathematics, Bengal Engineering and Science University, Shibpur, Howrah-711 103, India. \\ $\dagger$ Department of Mathematics, Jadavpur University, Kolkata-700 032, India. \\ Pacs no : 04.20.Dw, 04.20.Ex, 04.20.Cv, 04.70.Bw
}

\begin{abstract}
In this work, we have investigated the outcome of gravitational collapse in Husain space-time in the presence of electro-magnetic and a scalar field with potential. In order to study the nature of the singularity, global behavior of radial null geodesics have been taken into account. The nature of singularities formed has been thoroughly studied for all possible variations of the parameters. These choices of parameters has been presented in tabular form in various dimensions. It is seen that irrespective of whatever values of the parameters chosen, the collapse always results in a naked singularity in all dimensions. There is less possibility of formation of a black hole. Hence this work is a significant counterexample of the cosmic censorship hypothesis.
\end{abstract}

\section{Introduction}

When a massive star is on the verge of completing its nuclear cycle, then the thermonuclear reactions in the interior of the star cannot counter balance the immense gravitational pull of the star. Under most general conditions general relativity predicts that such a collapse must end in a singularity, which may or may not be clothed by an event horizon. A singularity may be physically described as a region in the space-time with extreme curvature, vanishing volume and unbounded gravitational forces. However, general relativity remains silent on the nature (BH or NS) or physical properties of such a singularity. This is basically due to the fact that mathematical structure breaks down preventing analysis at and beyond the singularity. This has triggered extensive research on Gravitational collapse during the past few decades. After all one would always like to know whether, and under what conditions gravitational collapse leads to the formation of a black hole $(\mathrm{BH})$. A few decades back R. Penrose (1969) proposed the cosmic censorship hypothesis (CCH), which states that the singularities formed in gravitational collapse of physically reasonable matter cannot be seen by any distant observer in the universe. It implies that the singularities formed in asymptotically flat space-times are always bounded by event horizons and hence are destined to be black holes. With the announcement of this proposal, study of gravitational collapse has gained special importance, because one would always like to know that whether there exists any physical collapse solutions that lead to naked singularities (NS), which will serve as counterexamples of $\mathrm{CCH}$.

Till date there has been a lot of significant work in gravitational collapse. The pioneering work of gravitational collapse appeared in the famous paper of Oppenheimer and Snyder (1939) in which they studied the gravitational collapse of adiabatically flowing dust. The outcome of the experiment led to the conclusion that the end state of collapse is a BH. Since then extensive research has been carried out to find more generalized results. But it took no less than 30 years since the Oppenheimer-Snyder model that the feasibility of NS was

\footnotetext{
${ }^{1}$ ujjal@iucaa.ernet.in, ujjaldebnath@yahoo.com

2 prudra.math@gmail.com

${ }^{3}$ biswas.ritabrata@gmail.com
} 
brought out by Christodoulou (1984) in his study of Lemaitre-Tolman-Bondi (LTB) model. It is thus understandable that, due to a lack of any alternative scenario all these years, the black hole became the unique end state of continued gravitational collapse for a remnant mass of a collapsing star beyond the threshold neutron star mass limit. The absence of analytical results led to several conjectures as well, namely, the (weak and strong) cosmic censorship conjecture (CCC) by Penrose, hoop conjecture (HC) by Thorne (1972), and Seifert (1979) conjecture, which to date remain unproven.

In order to study gravitational collapse properly, it is necessary to describe adequately the geometry of interior and exterior regions and to give conditions which allow the matching of these regions. In this context it is worthy to mention that collapse with static exterior was studied by Misner and Sharp (1964) for a perfect fluid in the interior. The concept of outgoing radiation in order to study the outcome of gravitational collapse was first used by Vaidya (1951). Later on Santos and collaborations (Santos, N.O. 1984; Oliveira, A.K.G. 1985,1987), Ghosh and Deskar $(2000,2003)$ and Cissoko et al (1998) have done meaningful work in gravitational collapse, thus extending the topic to new heights. The credit of studying gravitational collapse with a model of arbitrary shape (non-spherical) goes to Szekeres (1975) who in his model (known as Szekeres' model with perfect fluid (or dust) gave detailed solution of gravitational collapse for quasi-spherical or quasi-cylindrical symmetry of the space-time. Debnath et al (2005) explored gravitational collapse of the non-adiabatic fluid by assuming quasi-spherical Szekeres space-time in the interior and plane symmetric Vaidya solution in the exterior region. By using the local conservation of momentum, they studied the thermodynamical behavior of the collapsing matter. An extensive survey by Herrera (2004) and Mitra (2006) predicts that gravitational collapse is highly dissipative process. This showed that the effects of dissipation must be included in the study of gravitational collapse for its better understanding. Herrera and Santos (2004) then explored dynamical description of gravitational collapse by using Misner and Sharps formulation. Nolan (2002) investigated naked singularities in the cylindrical gravitational collapse of counter rotating dust shell. Di Prisco (2009) discussed the shear free cylindrical gravitational collapse by using junction conditions. Nakao (2009) studied gravitational collapse of a hollow cylinder composed of dust. Recently, Sharif and Rehmat (2010) discussed the dynamics of viscous dissipative plane symmetric gravitational collapse.

The general problem of gravitational collapse always remains intractable due to the complex and tedious form of the Einstein equations. Therefore instead of the general form of the equations, special forms are only considered to reach meaningful results. Two dimensional reduction of general relativity using spherical symmetry is one such case. Walking on this very path Husain in 1996, gave non-static spherically symmetric solutions of the Einstein equations for a null fluid source with pressure $p$ and density $\rho$ related by $p=k \rho$. The solution has a number of interesting features including BHs with short hair (Brown,J.D. and Husain,V. 1997). This solution is considered as a generalization of Vaidya solution, which was accomplished by Wang,A. and Wu,Y. (1999). Recently, Patil et al $(2005,2006)$ have studied the gravitational collapse of the Husain solution in four and five dimensional space-times.

In this paper we mainly propose to investigate the nature of singularity (BH or NS) formed by the collapse of a star in Husain metric accompanied by a electro-magnetic and a scalar field. In section (2) we present the basic equations for Husain solution in $(n+2)$ dimensional spherically symmetric space-time coupled with an electro-magnetic and a scalar field and we investigate the nature of singularity formed. We study the effect of accretion phenomenon on collapsing procedure in section (3). Finally, we end with some concluding remarks in section (4).

\section{Basic Equations for Husain Solution in $(n+2)$-Dimensional Spher- ically Symmetric Space-Time with Electro-Magnetic Field and Scalar Field}

Here we consider the metric in $(n+2)$-dimensional spherically symmetric space-time in the form

$$
d s^{2}=-\left(1-\frac{m(v, r)}{r^{n-1}}\right) d v^{2}+2 d v d r+r^{2} d \Omega_{n}^{2}
$$

where $r$ is the radial co-ordinate and $v$ is the null co-ordinate, $m(v, r)$ gives the gravitational mass inside the sphere of radius $r$ and $d \Omega_{n}^{2}$ is the line element on a unit $n$-sphere. 
The Einstein's field equation for matter field, electro-magnetic field and scalar field with cosmological constant $(\Lambda)$ has the following form (choosing $8 \pi G=c=1$ )

$$
G_{\mu \nu}=T_{\mu \nu}+E_{\mu \nu}+H_{\mu \nu}-\Lambda g_{\mu \nu}
$$

Now we consider two types of fluids like Vaidya null radiation and a perfect fluid having the form of the energy momentum tensor

$$
T_{\mu \nu}=T_{\mu \nu}^{(n)}+T_{\mu \nu}^{(m)}
$$

with

$$
T_{\mu \nu}^{(n)}=\sigma l_{\mu} l_{\nu}
$$

and

$$
T_{\mu \nu}^{(m)}=(\rho+p)\left(l_{\mu} \eta_{\nu}+l_{\nu} \eta_{\mu}\right)+p g_{\mu \nu}
$$

Where, $\rho$ and $p$ are the energy density and pressure for the perfect fluid and $\sigma$ is the energy density corresponding to Vaidya null radiation. In the co-moving co-ordinates $\left(v, r, \theta_{1}, \theta_{2}, \ldots, \theta_{n}\right)$, the two eigen vectors of energy-momentum tensor namely $l_{\mu}$ and $\eta_{\mu}$ are linearly independent future pointing null vectors having components

$$
l_{\mu}=(1,0,0, \ldots, 0) \text { and } \eta_{\mu}=\left(\frac{1}{2}\left(1-\frac{m}{r^{n-1}}\right),-1,0, \ldots, 0\right)
$$

and they satisfy the relations

$$
l_{\lambda} l^{\lambda}=\eta_{\lambda} \eta^{\lambda}=0, l_{\lambda} \eta^{\lambda}=-1
$$

The energy- momentum tensor $E_{\mu \nu}$ of the electro-magnetic field is given by

$$
E_{\mu \nu}=\frac{1}{4 \pi}\left[F_{\mu}^{\nu} F_{\mu \nu}-\frac{1}{4} g_{\mu \nu} F^{\nu \delta} F_{\nu \delta}\right]
$$

where $F_{\mu \nu}$ is the electro-magnetic field tensor satisfying Maxwell's equations

$$
F_{\mu \nu}=\psi_{\nu, \mu}-\psi_{\mu, \nu}
$$

and

$$
\frac{1}{\sqrt{-g}} \partial_{\mu}\left(\sqrt{-g} F^{\mu \nu}\right)=-4 \pi J^{\nu}
$$

where $\psi_{\mu}$ is the four-potential and $J_{\mu}$ is the four-current.

Since the charge is at rest in this system, so there will be no magnetic field in this system. Thus, we may choose the four-potential and four-current as

$$
\psi_{\mu}=(\psi(v, r), 0,0,0)
$$

and

$$
J^{\mu}=\tau l^{\mu}
$$

where $\tau$ is the charge density.

The energy-momentum tensor $H_{\mu \nu}$ for the scalar field $\phi(v, r)$ driven by the potential $V(\phi)$ is given by

$$
H_{\mu \nu}=\phi,_{\mu} \phi,_{\nu}-\frac{1}{2} g_{\mu \nu} g^{\alpha \beta} \phi,_{\alpha} \phi,_{\beta}-g_{\mu \nu} V(\phi)
$$

with

$$
\frac{1}{\sqrt{-g}} \partial_{\mu}\left(\sqrt{-g} \partial^{\mu} \phi\right)=-\frac{d V(\phi)}{d \phi}
$$

From equations (9) - (12), we have the only non-zero component of the field tensor as

$$
F_{01}=-F_{10}=-\psi^{\prime}=-\frac{e(v)}{r^{n}}
$$

where $e(v)$ is the arbitrary function of $v$. 
From Einstein's field equation (2), we have

$$
\begin{gathered}
\sigma=\frac{n \dot{m}}{2 r^{n}}-\dot{\phi}^{2} \\
\rho=\frac{n m^{\prime}}{2 r^{n}}-\frac{e^{2}(v)}{8 \pi r^{2 n}}-V(\phi)-\Lambda \\
p=-\frac{m^{\prime \prime}}{2 r^{n-1}}-\frac{e^{2}(v)}{8 \pi r^{2 n}}+V(\phi)+\Lambda \\
V(\phi)=\frac{E(v)}{r}+D(r)
\end{gathered}
$$

where an over-dot and dash stand for differentiation with respect to $v$ and $r$ respectively and $E(v)=-\int \dot{\phi}^{2} d v$ and $D(r)$ is an arbitrary integration function.

We assume the matter fluid obeys the barotropic equation of state

$$
p=k \rho, \quad(k, \text { a constant })
$$

Now using (17), (18) and (20) in the Einstein field equations, we have the differential equation in $m$,

$$
r^{2} m^{\prime \prime}+k n r m^{\prime}=\frac{(k-1) e^{2}(v)}{4 \pi r^{n-1}}+2(k+1)\left[\frac{E(v)}{r}+D(r)+\Lambda\right] r^{n+1}
$$

Let us choose $D(r)=D_{0} r^{\beta}$, for which the explicit solution for $m$ is $(n k \neq 1)$

$$
m(v, r)=f(v)-\frac{g(v)}{(n k-1) r^{n k-1}}-\frac{e^{2}(v)}{4 \pi n(n-1) r^{n-1}}+\frac{2 \Lambda r^{n+1}}{n(n+1)}+\frac{2(k+1) E(v) r^{n}}{n(n k+n-1)}+\frac{2(k+1) D_{0} r^{\beta+n+1}}{(\beta+n+1)(\beta+n k+n)}
$$

Therefore the solution of the Einstein equations with matter field, electro-magnetic field and scalar field given by equation (2) can be written as

$$
\begin{aligned}
d s^{2}=-[1 & -\frac{f(v)}{r^{n-1}}+\frac{g(v)}{(n k-1) r^{n(k+1)-2}}+\frac{e^{2}(v)}{4 \pi n(n-1) r^{2(n-1)}}-\frac{2 \Lambda r^{2}}{n(n+1)} \\
& \left.-\frac{2(k+1) E(v) r}{n(n k+n-1)}-\frac{2(k+1) D_{0} r^{\beta+2}}{(\beta+n+1)(\beta+n k+n)}\right] d v^{2}+2 d v d r+r^{2} d \Omega_{n}^{2}
\end{aligned}
$$

This is termed as generalization of Husain solution in higher dimension.

We shall discuss the existence of NS in generalized Vaidya space-time by studying radial null geodesics. In fact, we shall examine whether it is possible to have outgoing radial null geodesics which were terminated in the past at the central singularity $r=0$. The nature of the singularity (NS or $\mathrm{BH}$ ) can be characterized by the existence of radial null geodesics emerging from the singularity. The singularity is at least locally naked if there exist such geodesics and if no such geodesics exist it is a $\mathrm{BH}$.

The equation for outgoing radial null geodesics can be obtained from equation (11) by putting $d s^{2}=0$ and $d \Omega_{n}^{2}=0$ as

$$
\frac{d v}{d r}=\frac{2}{\left(1-\frac{m(v, r)}{r^{n-1}}\right)} .
$$

It can be seen easily that $r=0, v=0$ corresponds to a singularity of the above differential equation. Suppose $X=\frac{v}{r}$ then we shall study the limiting behavior of the function $X$ as we approach the singularity at $r=0, v=0$ along the radial null geodesic. If we denote the limiting value by $X_{0}$ then

$$
\begin{aligned}
& X_{0}=\lim _{v \rightarrow 0} X \quad \lim _{v \rightarrow 0} \frac{v}{r}=\lim _{v \rightarrow 0} \frac{d v}{d r}=\lim _{v \rightarrow 0} \frac{2}{\left(1-\frac{m(v, r)}{r^{n-1}}\right)} \\
& \begin{array}{rlll}
r \rightarrow 0 & r \rightarrow 0 & r \rightarrow 0 & r \rightarrow 0
\end{array}
\end{aligned}
$$


Table: The table represents the values of $X_{0}$ for different values of parameters in various dimensions.

\begin{tabular}{|c|c|c|c|c|c|c|c|c|c|c|}
\hline \multirow{2}{*}{$\mathrm{f}_{0}$} & \multirow[b]{2}{*}{$\mathrm{g}_{0}$} & \multirow[b]{2}{*}{$\mathrm{e}_{0}$} & \multirow{2}{*}{$\mathrm{E}_{0}$} & \multicolumn{7}{|c|}{$\mathrm{X} 0$} \\
\hline & & & & $\mathrm{n}=2$ & $\mathrm{n}=3$ & $\mathrm{n}=4$ & $=5$ & $\mathrm{n}=6$ & $\mathrm{n}=7 . \quad \mathrm{r}$ & $\mathrm{n}=8$ \\
\hline 0.2 & 0.5 & 1 & 1 & 1.4478 & 1.3999 & 1.3563 & 1.320 & 1.2902 & 1.2653 & 1.244 \\
\hline .002 & 0.5 & 1 & 1 & 1.3480 & 1.2986 & 1.26241 & 1.235 & 1.2124 & 1.1942 & 1.179 \\
\hline 5 & 0.5 & 1 & 1 & & 4.3469 & 3.06494 & 2.494 & & 1.9712 & 1.829 \\
\hline 0.5 & .002 & 1 & 1 & 10.236 & 5.8129 & 4.07263 & 3.2452 & 2.7706 & 2.4645 & 2.25 \\
\hline 0.5 & 8 & 1 & 1 & 18 & 0.8444 & 0.92 & 0.9711 & 0.9 & & 1.023 \\
\hline 0.5 & 0.5 & .001 & 1 & 1.6875 & 1.6169 & 1.53907 & 1.473 & 1.4203 & 1.378 & 1.343 \\
\hline 0.5 & 0.5 & 5 & 1 & 1.1332 & 1.2857 & 1.3 & 1.323 & & & 1.280 \\
\hline 0.5 & 0.5 & 0.5 & 0.001 & 1.4669 & 1.5596 & 1.5192 & 1.464 & 1.4 & 1.3753 & 1.341 \\
\hline 0.5 & 0.5 & 0.5 & 5 & & & 1.58963 & 1.494 & 1.43006 & 1.3830 & 1.346 \\
\hline 5 & 5 & 5 & 5 & 1.2611 & & & 1.401 & 1.36974 & 1.3406 & 1.314 \\
\hline .00 & 005 & .005 & .005 & .9839 & 1.9659 & & 1.823 & & & 1.569 \\
\hline .005 & 0.005 & 10 & 0.005 & 0.6910 & 0.9542 & 1.05294 & 1.096 & 1.1163 & 1.125 & 1.129 \\
\hline .005 & 0.005 & .005 & 10 & 7.1170 & 3.524 & 2.52932 & .1000 & 1.86533 & 1.7174 & 1.615 \\
\hline
\end{tabular}

Using (22) and (25), we have

$$
\begin{aligned}
& \frac{2}{X_{0}}=\lim _{\substack{v \rightarrow 0 \\
r \rightarrow 0}}\left[1-\frac{f(v)}{r^{n-1}}+\frac{g(v)}{(n k-1) r^{n(k+1)-2}}+\frac{e^{2}(v)}{4 \pi n(n-1) r^{2(n-1)}}-\frac{2 \Lambda r^{2}}{n(n+1)}\right. \\
& \left.-\frac{2(k+1) E(v) r}{n(n k+n-1)}-\frac{2(k+1) D_{0} r^{\beta+2}}{(\beta+n+1)(\beta+n k+n)}\right]
\end{aligned}
$$

Now choosing, $f(v)=f_{0} v^{n-1}, g(v)=g_{0} v^{n(k+1)-2}, e(v)=e_{0} v^{n-1}$ and $E(v)=\frac{E_{0}}{v}$, we obtain the algebraic equation of $X_{0}$ as

$$
f_{0} X_{0}^{n}-\frac{g_{0}}{n k-1} X_{0}^{n k+n-1}-\frac{e_{0}^{2}}{4 \pi n(n-1)} X_{0}^{2 n-1}+\frac{2(k+1) E_{0}}{n(n k+n-1)}-X_{0}+2=0
$$

From above equation, we see that the equation is completely independent of $\Lambda$ and $D_{0}$.

Now if we get only non positive solution of the equation we can assure the formation of a BH. Getting a positive root indicates a chance to get a NS. Since the obtained equation is an highly complicated one, it is extremely difficult to find out an analytic solution of $X_{0}$ in terms of the variables involved. So our idea is to find out different numerical solutions of $X_{0}$, by assigning particular numerical values to the associated variables.

The different solutions of $X_{0}$ for different sets of parametric values $\left(f_{0}, g_{0}, e_{0}, E_{0}\right)$ are given here in a tabular form. It is seen that over a large range of parametric values the positive solution for $X_{0}$ is the only outcome, which immediately implies the occurrence of NS as the outcome of the collapse. 


\section{Impact of Accretion upon the collapsing body}

The highly massive star, white dwarf or the other things which are going to collapse to form a singularity they do so as their mass limit crosses the Chandrasekhar's limit. In general cases it happens as the object inhells mass from the dust cloud nearby or from a companion star which is in a binary system with the concerned object. Collapse and accretion together has been studied in literature since a long time(Wagh, S.M. 2002; Vorobyov,E.I. and Basu,S. 2005; Johnson,J.L. 2010).

Now if we assume that the object we are concerning is collapsing with an accretion procedure together then we can modify our mass function to the expression given by

$$
M-\dot{M} d v=M-\Omega_{n} r^{n} T_{0}^{1} d v
$$

The $T_{0}^{1}$ represents the non-diagonal stress energy tensor component. The explicit form of $T_{0}^{1}$ is given by the equations (3), (4) and (5). Using which we can calculate the value of $T_{0}^{1}$ and the modified form of the $f(r, v)$ represented by a $\bar{f}(r, v)$ has the expression

$$
\bar{f}(r, v)=f(r, v)+\frac{\Omega_{n} \sigma}{r^{n}}
$$

From which with the assumption $\sigma=\sigma_{0} v$ the equation of $X_{0}$ comes as

$$
f_{0} X_{0}^{n}-\frac{g_{0}}{n k-1} X_{0}^{n k+n-1}-\frac{e_{0}^{2}}{4 \pi n(n-1)} X_{0}^{2 n-1}-\frac{\pi^{\frac{n}{2}} \sigma_{0}}{\Gamma\left(1+\frac{n}{2}\right)} X_{0}^{2}+\frac{2(k+1) E_{0}}{n(n k+n-1)}-X_{0}+2=0
$$

Here again the analytical solution of the equation is bit tough. Rather we can solve $X_{0}$ easily for different parametric values. But after going through this procedure we found that $X_{0}$ does not possess any values in the negative region. Hence this result derives us into plotting graphs for $X_{0}$, in various regions in order to get an explicit idea of the nature of solution of the concerned equation. After plotting $X_{0}$ with different ranges of all other parameters it is quite clear from the graphs that $X_{0}$ always has a positive value for any combination of the other parameters. So even if accretion procedure is going upon the collapsing object the chance of having NS always will be there.

\section{Conclusion}

Here we have considered the $(n+2)$-dimensional Husain space-time in presence of radiating null fluid, barotropic fluid, an electro-magnetic field and a scalar field with potential. The corresponding solutions have been obtained. Next we have analyzed the gravitational collapse of a star in Husain space-time. The existence of outgoing radial null geodesic has been thoroughly investigated in order to characterize the nature of central singularity formed as the end state of collapse. Equation of radial null geodesic is formed and the values of the variable $X_{0}$ is computed for different values of parameters in different dimensions. Surprisingly in all the cases it was found that irrespective of the values of the parameters chosen, the value of $X_{0}$ is always positive and hence the singularity formed is always a naked singularity. This has been shown in a tabular form. Impact of accretion phenomenon on the gravitational collapse of a star has also been taken into account. The mass function for a collapsing procedure is considered and from it, the equation of $X_{0}$ is constructed for various dimensions. To study this case graphs were drawn for different values of the parameters $\left(f_{0}, g_{0}, e_{0}, E_{0}\right)$ and it was found that the figures did not show any tendency of sneaking into the negative region. Hence black holes may not be the correct option for Husain metric in any dimension. Formation of event horizons is totally barred in Husain metric. So the only possibility is a naked singularity. Hence this is a significant counter example of the cosmic censorship hypothesis.

\section{Acknowledgement :}

RB thanks West Bengal State Govt. for awarding SRF. 

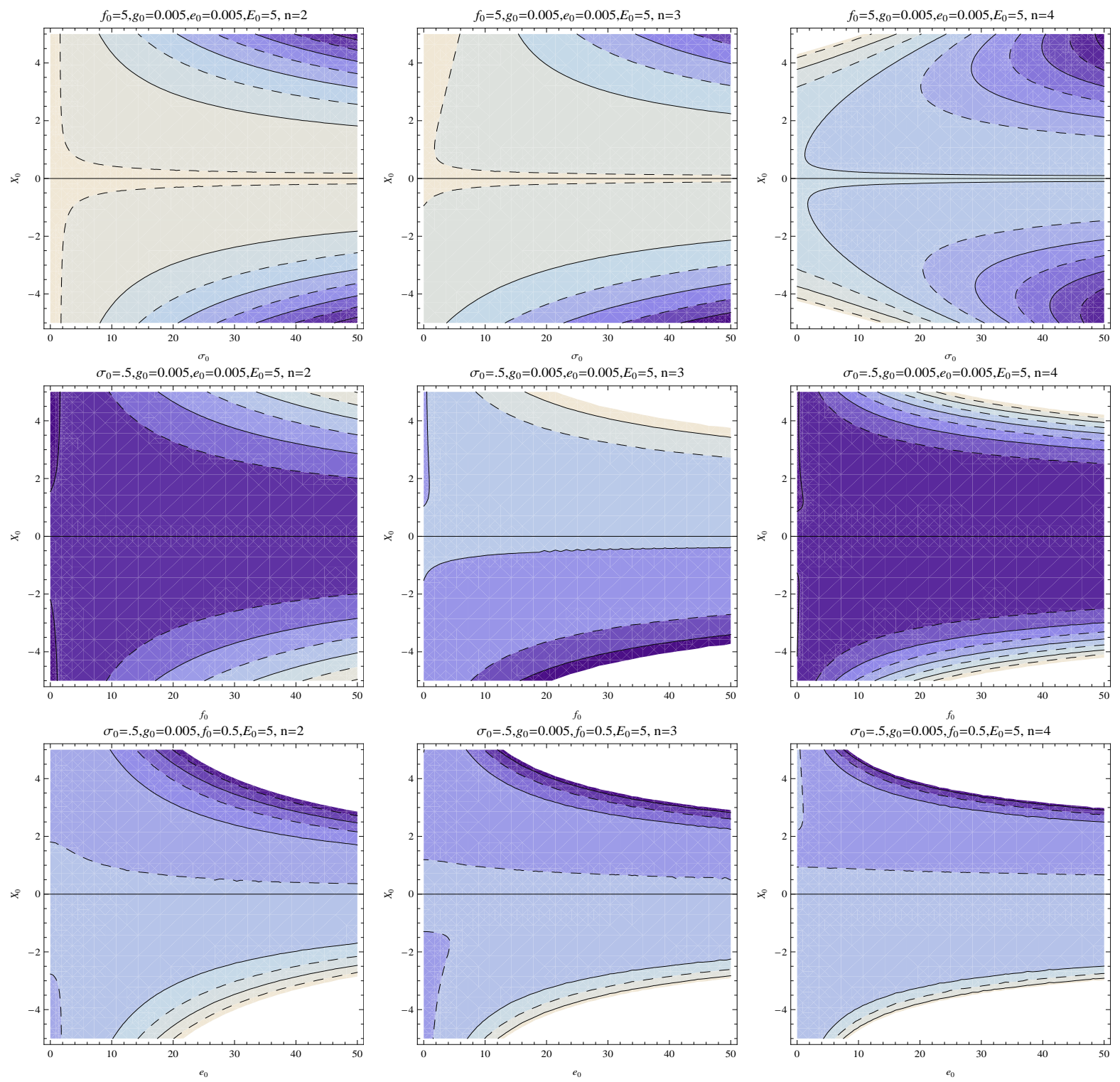

The figures represent the plot of $X_{0}$ (30) with the variation of different parameters in various dimensions when some fluids are accreting upon collapsing objects. 

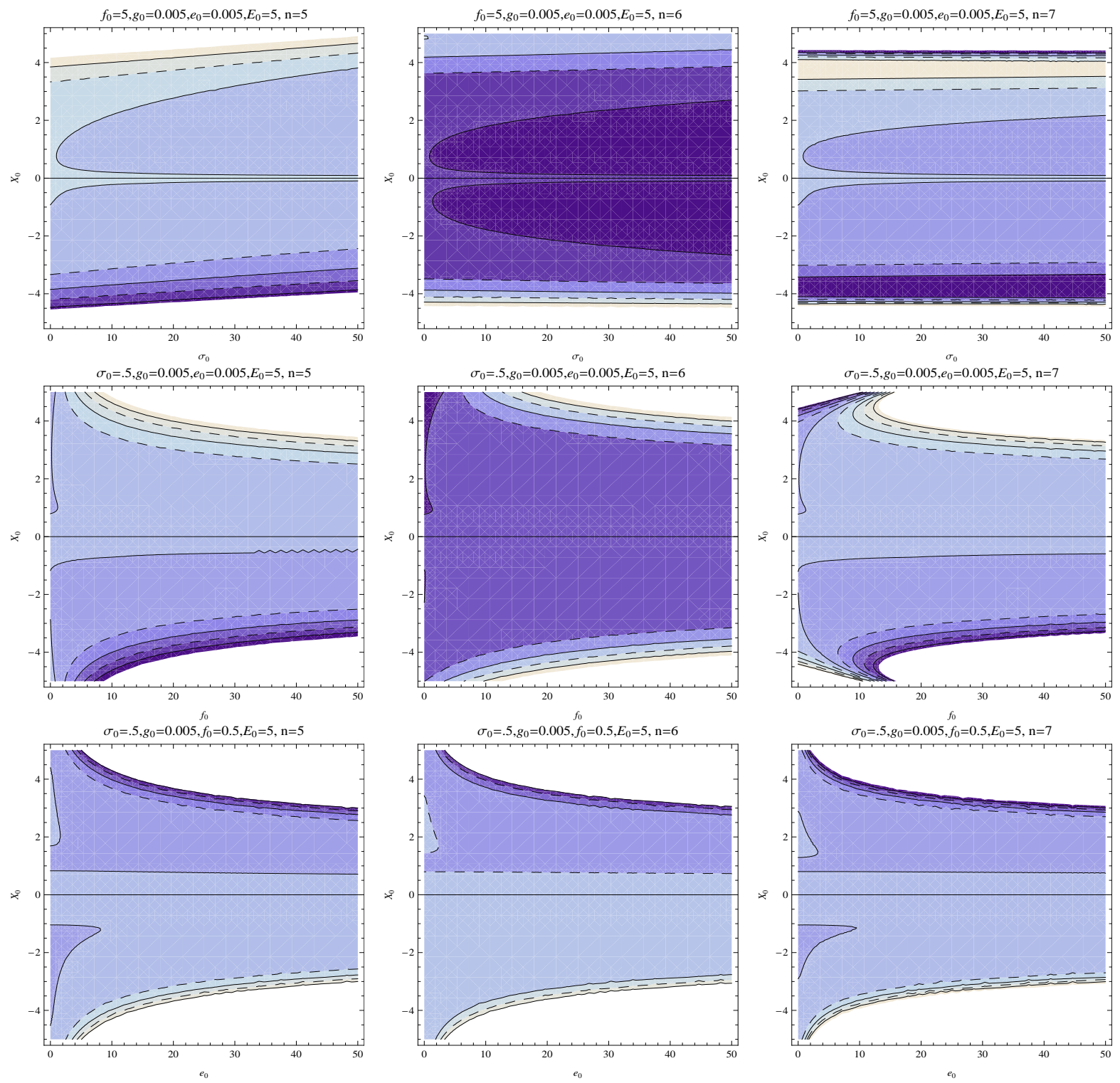

The figures represent the plot of $X_{0}(\underline{30})$ with the variation of different parameters in various dimensions. 


\section{References}

Brown, J. D., . Husain, V :- Int. J. Mod. Phys. D 6563 (1997).

Christodoulou, D. :-Commun. Math. Phys 93, 171 (1984).

Cissoko,M., Fabris, J.,Gariel, J. ,Denmat, G. L.,Santos, N. O. :- $g r-q c / 9809057$.

Debnath, U., Nath, S., Chakraborty, S.:- Gen. Relativ.grav 37, 215(2005).

Di Prisco, A., Herrera, L., Denmat, G.Le., MacCallum,M.A.H., Santos, N.O.:- Phys. Rev. D80, 064031(2009).

Ghosh, S. G., Deshkar,D. W. :- Gravitation and Cosmology 61 (2000).

Ghosh, S. G. , Deshkar, D. W.:- Int. J. Mod. Phys. D12 317 (2003).

Herrera, L., Santos, N.O.: -Phys. Rev. D70, 084004(2004).

Herrera, L., Di Prisco, A., Martin, J. Ospino, J. Santos,N.O., Troconis, O.:-Phys. Rev. D69, 084026(2004).

Husain, V. :- Phys. Rev. D 53 R1759 (1996).

Johnson,J.L., Sadegh Khochfar, Thomas H. Greif, Fabrice Durier,. arXiv:1007.3849 [astro-ph.CO]

Misner, C. W. , Sharp, D. :- Phys. Rev. 136 b571 (1964).

Mitra, A.:- Phys. Rev. D74, 024010(2006).

Nakao, K., Harada, T., Kurita, Y., Morisawa, Y.:- Prog.Theor. Phys. 122, 521(2009).

Nolan, B.C.:- Phys. Rev. D65, 104006(2002).

Oliveira, A. K. G. de,Santos,N. O., Kolassis,Mon. Not. R. Astr. Soc. 216 1001(1985).

Oliveira, A. K. G. de, Santos, N. O. :-Astrophys. J.312 640 (1987).

Oppenhiemer,J. R., Snyder, H. :- Phys. Rev. 56455 (1939).

Patil,K. D. , Thool, U. S. :-Int. J. Mod. Phys. D 14873 (2005).

Patil, K. D., Zade, S. S. :- Int. J. Mod. Phys. D 151359 (2006).

Penrose, R:-Riv Nuovo. Cimento. 1, 252 (1969).

Santos,N. O. :- Phys. Lett. A 106296 (1984).

Seifert, H. J. :- Gen. Relativ. Gravit. 10, 1065 (1979).

Sharif, M., Rehmat, Z.:-Gen. Relativ. Grav.42,1795(2010).

Szekeres, P. :-Phys. Rev. D 122941 (1975).

Thorne, K. S. :-in Magic without Magic, edited by J. R.Klander (Freeman, San Francisco, 1972).

Vaidya, P. C. :-Proc. Indian Acad. Sci. A 33264 (1951).

Vorobyov,E.I., Basu,S.,:- MNRAS $360675(2005)$.

Wagh, S. M.:-arXiv astro-ph/0202054.

Wang, A., Wu,Y. :- Gen. Rel. Grav. 31107 (1999). 